\title{
Anaesthetic Efficacy and Physiological Response of Clove Oil and Lidocaine-HCl on River Puffer, Takifugu obscurus and Tiger Puffer, T. rubripes
}

\author{
${ }^{\dagger}$ In-Seok Park \\ Division of Marine Bioscience, College of Ocean Science and Technology, Korea Maritime \& Ocean University, \\ Busan 49112, Korea
}

\begin{abstract}
The effects of the anaesthetic agents, clove oil and mixture of clove oil with lidocaine-HCl were evaluated on river puffer, Takifugu obscurus and tiger puffer, T. rubripes. Anaesthesia times of clove oil were affected by water temperature $\left(20^{\circ} \mathrm{C}, 24^{\circ} \mathrm{C}\right.$, and $\left.28^{\circ} \mathrm{C}\right)$ and salinity $(10,20$, and $30 \mathrm{ppt})$. Anaesthesia times of mixed samples were significantly similar with regard to exposure and recovery times, and all samples satisfied anaesthesia criteria (exposure time within 3 min and recovery time within $5 \mathrm{~min}$ ) under the various temperatures and salinities, and the lowest to highest concentration of anaesthetics $(p<0.05)$. Both species river puffer and tiger puffer had short exposure time with a high anaesthesia dose, high temperature $\left(28^{\circ} \mathrm{C}\right)$ and intermediate salinity $(20 \mathrm{ppt})$, and were highly affected by temperature and salinity $(p<0.05)$. The mixed anaesthetics had rapid exposure times and long recovery times in contrast to the effects of clove oil. Cortisol concentrations under the conditions of various clove oil dosages, salinity, and temperature for both species increased until 12 hrs after recovery from anaesthesia $(p<0.05)$. After $12 \mathrm{hrs}$, cortisol concentrations decreased until after $48 \mathrm{hrs}(p<0.05)$. During the simulated transportation of both species, control and sedated clove oil groups (5 ppm) were measured for water parameters, dissolved oxygen (DO), $\mathrm{CO}_{2}$, respiratory frequency, $\mathrm{NH}_{4}{ }^{+}$, and $\mathrm{pH}$ for $6 \mathrm{hrs}$ in $1 \mathrm{hr}$ intervals. Water parameters of sedated groups and controls were significantly different after 2 hrs $(p<0.05)$.
\end{abstract}

Key words : Clove oil, Lidocaine-HCl, River puffer, Plasma cortisol, Tiger puffer, Transportation

\section{INTRODUCTION}

Anaesthetic agents have been used in fish handling situations primarily when there is a need for rapid processing, such as transporting, inhibiting fish activities, culturing, tagging, measuring, injecting vaccines and antibacterial substances, medical treatment for diseases, artificial spawning, sorting, and preparation for trade (Bell, 1987; Barton \& Iwama, 1991; Westerfield, 1993; Munday \& Wilson,
1997; Park et al., 1998a, 2017a; Mommsen et al., 1999; Pirhonen \& Schreck, 2003; Gil et al., 2016). These processing activities may result in negative behavioural and physiological effects such as decreased feeding, inhibition or enhancement of aggressive behaviour and susceptibility to disease (Ross \& Ross, 2008). The use of anaesthetics during the handling procedures provides a way to minimize these deleterious effects, to immobilize fish for some time, and to reduce physical damage that might occur dur-

Manuscript received December 29, 2018, Received in revised form January 17, 2019, Accepted February 16, 2019

${ }^{\dagger}$ Corresponding Author : In-Seok Park, Division of Marine Bioscience, College of Ocean Science and Technology, Korea Maritime \& Ocean University, Busan 49112, Korea. Tel: +82-51-410-4321, Fax: +82-51-404-4750, E-mail: ispark@kmou.ac.kr

This is an Open Access article distributed under the terms of the Creative Commons Attribution Non-Commercial License (http:// creative-commons.org/licenses/by-nc/3.0) which permits unrestricted non-commercial use, distribution, and reproduction in any medium, provided the original work is properly cited. 
ing handling activities (Park et al., 1998b, 2017a; Ross \& Ross, 2008; Gil et al., 2016). Anaesthesia also has no harmful effect on mortality and morbidity (Cooke et al., 2004).

Recently, anaesthetics used in aquaculture have satisfied several conditions such as safety to both user and fish, affordability in price, nontoxicity to the environment, and no withdrawal period with synthetic-based anaesthetics like MS-222 (Pirhonen \& Schreck, 2003; Park et al., 2017a). Clove oil is used as a food additive and lidocaine$\mathrm{HCl}$ is used as a local anaesthetic agent in dentistry. Both are safe for fish and humans (Park et al., 2011, 2017a, 2017b). Thus, clove oil and lidocaine- $\mathrm{HCl}$ [2-(diethylamino)$\mathrm{N}$-(2, 6-dimethylphenyl) acetimide hydrochloride] have become more and more popular in the aquaculture industry (Bell, 1987; Park et al., 2011). The anaesthetic effects of clove oil and lidocaine- $\mathrm{HCl}$ have been found to be excellent in a number of fish species (Park et al., 2011, 2017a). The anaesthesia exposure time is within $3 \mathrm{~min}$ or less and the recovery time is within 5 min or less (Summerfelt \& Smith, 1990; Woolsey et al., 2004). Various concentrations of clove oil and lidocaine- $\mathrm{HCl}$ which were used to anaesthetize a variety of samples gave satisfactory exposure times and recovery times regardless of the size and weight of the sample (Park et al., 2011, 2017a, 2017b).

Fish transportation is a very important part of the aquaculture industry and it is essential to minimize the physical stress on the fish during transportation (Cooke et al., 2004). Sedative effects of anaesthesia are different from direct anaesthesia in that a low anaesthesia concentration will slow down the metabolic activity of fish, which is beneficial when transporting fish (Ross \& Ross, 2008; Gil et al., 2016; Park et al., 2017a).

The river puffer, Takifugu obscurus and tiger puffer, Takifugu rubripes are cannibalistic species that have been regarded as important aquaculture species in Japan and Korea. Moreover, river puffer and tiger puffer have an economic advantage of high value resulting from their excellent flesh quality (Park et al., 2017b). They bring in more than 2 times the price of olive flounder, Paralichthys olivaceus, rockfish, Sebastes zonatus, yellowtail, Seriola quinqueradiata, or red seabream, Pagrus major (Kikuchi et al., 2007). However, river puffer and tiger puffer have sharp teeth with which they can cut nets or cannibalize each other, so that sedation during the transport is economically advantageous (Kang et al., 2005). Therefore, the aim of this study is to determine the optimum concentrations of anaesthetic clove oil and the mixture of two anaesthetics, clove oil and lidocaine- $\mathrm{HCl}$ for anaesthetizing river puffer and tiger puffer with a range of salinity and temperatures.

\section{MATERIALS AND METHODS}

\section{Experimental fish}

In 2018, 96 specimens each of river puffer and tiger puffer were purchased from puffer fish hatchery in Korea. The samples were transported to and reared in the Fishery Genetics and Breeding Sciences Laboratory of the Korea Maritime and Ocean University, Busan, Korea. The initial measurements of body weight (BW) and total length (TL) were made to the nearest $0.1 \mathrm{~g}$ and $0.1 \mathrm{~cm}$ using an electric balance (AX 200, Shimadzu Corp., Japan) and digital vernier calipers (CD-20 CP, Mitutoyo, Japan) respectively. The TL and BW of each species were $13.7 \pm 2.24 \mathrm{~cm}$ and $47.5 \pm 4.27 \mathrm{~g}$ for river puffer, and $15.2 \pm 2.67 \mathrm{~cm}$ and $56.2 \pm 6.35 \mathrm{~g}$ for tiger puffer respectively. Experimental samples were acclimated for one week in aquariums with different water temperatures $\left(20^{\circ} \mathrm{C}, 24^{\circ} \mathrm{C}\right.$, and $\left.28^{\circ} \mathrm{C}\right)$ for sufficient adaptation and all samples were starved for 24 hrs prior to the experiments.

\section{Anaesthetic effect criteria}

As shown in Table 1, exposure and recovery behavior stages are categorized in accordance with the anaesthetic effect (Summerfelt \& Smith, 1990; Woolsey et al., 2004). Exposure time is defined as from the time when the sam- 
Table 1. Stages of anaesthesia and recovery in a clove oil and lidocaine-HCl test performed on the river puffer, Takifugu obscurus and tiger puffer, T. rubripes*

\begin{tabular}{|c|c|}
\hline Stage & Characteristic behavior \\
\hline \multicolumn{2}{|c|}{ Anaesthesia } \\
\hline A1 & $\begin{array}{l}\text { Normal swimming; operculum movement and } \\
\text { normal general movement }\end{array}$ \\
\hline A2 & Swimming speed slowed; rolling from side to side \\
\hline A3 & Partial loss of equilibrium; swimming erratically \\
\hline A4 & $\begin{array}{l}\text { Complete loss of equilibrium; swimming perfectly } \\
\text { inside-out; pectoral fin, pelvic fin and dorsal fin } \\
\text { movement stop }\end{array}$ \\
\hline A5 & Little sedation; anal fin and tail fin movement stop \\
\hline A6 & Perfect sedation; only operculum movement \\
\hline A7 & Operculum movement ceased \\
\hline
\end{tabular}

Recovery

R1 Resume operculum movement

R2 Preferential movement of pectoral fin and tail fin

R3 Dorsal fin, pelvic fin, and anal fin movement

R4 Swimming perfectly inside-out

R5 Swimming erratically; redressing the balance

R6 Normal swimming; responsiveness to visual stimuli

${ }^{*}$ Modified from Summerfelt \& Smith (1990) and Woolsey et al. (2004).

ple was stocked in water containing anaesthetic to the time of reaching the A5 stage (Table 1; little sedation; anal fin and tail fin movement stops). Recovery time is defined as from the time when the fish were stocked in recovery water to the time of attaining the R6 stage, in which swimming and responsiveness to visual stimulation is normal (Table 1).

\section{Investigation of the anaesthetic effect}

The anaesthetic effect of clove oil (82\%-87\% eugenol:
Sigma, MO, USA) was investigated at four concentrations $(15,30,45$, and $60 \mathrm{ppm})$, three water salinities $(10,20$, and $30 \mathrm{ppt})$ and three temperatures $\left(20^{\circ} \mathrm{C}, 24^{\circ} \mathrm{C}\right.$, and $\left.28^{\circ} \mathrm{C}\right)$. The anaesthetic effect of the combination of two anaesthetics, clove oil and lidocaine-HCl (Sigma, MO, USA) was examined at three concentrations of clove oil $(15,30$, and $45 \mathrm{ppm})$ and three concentrations of lidocaine- $\mathrm{HCl}(400$, 500 , and $600 \mathrm{ppm})$ at $24^{\circ} \mathrm{C}$ and $30 \mathrm{ppt}$. Fish were exposed to different combinations of salinity, water temperature and anaesthesia concentrations. The stock solution clove oil was dissolved in 95\% ethanol (Sigma, MO, USA) at a ratio of 1:10 (Cho \& Heath, 2000). Low doses of ethanol have no anaesthetic effect on fish (Munday \& Wilson, 1997). The anaesthetic solution of lidocaine- $\mathrm{HCl}$ was neutralized with 1,000 ppm $\mathrm{NaHCO}_{3}$ (Park et al., 1998b), which was prepared as the total concentration to anaesthetic effect.

Exposure time was measured from the time when samples were transferred from a stock tank to an anaestheticcontaining aquarium $(30 \mathrm{~L})$ to when they reached A5 (Table 1) behavior; recovery time was measured between the time when the anaesthetized samples were transferred to a sea water tank containing $30 \mathrm{~L}$ with sufficient aeration and the time they reached R6 (Table 1) behavior.

\section{Water parameter in anaesthetic experiment}

Analyzed water quality parameters included the dissolved oxygen (DO), $\mathrm{CO}_{2}$, respiratory frequency, $\mathrm{NH}_{4}{ }^{+}$, and $\mathrm{pH}$ for $6 \mathrm{hrs}$ (pre, 1, 2, 3, 4, 5, and $6 \mathrm{hrs}$ ). To prevent sample mortality in the experiment, samples were processed under the diversified concentrations of clove oil for several hours, resulting in the concentration of clove oil for sedative action being $5 \mathrm{ppm}$. Prior to measuring DO, the $\mathrm{NH}_{4}{ }^{+}$and $\mathrm{CO}_{2}$ concentrations were measured using a spectrophotometer (DR 2800, Colorado, USA). The respiration frequency (gill cover movement) of fish was measured using a counter and a digital timer. The DO concentration was measured with an $\mathrm{O}_{2}$ electrode and a multi-data logger system (Oxyguard, Denmark). 


\section{Physiological response with the elapsed time af-} ter anaesthesia

The blood physiological response was measured at various times after the fish were anaesthetized with various clove oil concentrations $(15,30,45$, and $60 \mathrm{ppm})$, three water salinities $(10,20$, and $30 \mathrm{ppt})$ and three temperatures $\left(20^{\circ} \mathrm{C}, 24^{\circ} \mathrm{C}\right.$, and $\left.28^{\circ} \mathrm{C}\right)$. Blood samples were extracted from fish at 0 (pre), 1, 6, 12, 24, and $48 \mathrm{hrs}$.

In order to investigate the stress response of fish, blood of each sample was collected from the caudal vasculature using a disposable syringe $(3 \mathrm{~mL}$, Sung Shim Medical, Korea) with heparin sodium (Sigma, MO, USA) within 1 min after anaesthetic recovery. Collected blood was centrifuged at $200 \times \mathrm{g}$ for $10 \mathrm{~min}$ (Micro 17R, Hanil Science Industrial, Korea) and stored at $-80^{\circ} \mathrm{C}$ (Nihon Freezer, Japan) until plasma cortisol was analyzed. The collected plasma was transferred to another $1.5 \mathrm{~mL}$ microtube and stored at $-80^{\circ} \mathrm{C}$. The plasma cortisol concentration was determined with a cortisol radioimmunoassay kit (Coat-ACount TKCO Cortisol RIA Kit, DPC, USA).

\section{Statistical analysis}

One and two-way analysis of variance (ANOVA) were used to identify the significance $(p<0.05)$ of all triplicate experiments. The differences among groups were analyzed by ANOVA using the SPSS statistics package (SPSS 9.0, SPSS, Chicago, IL, USA), and multiple comparisons were performed using Duncan's multiple range test (Duncan, 1955).

\section{RESULTS}

\section{Investigation of the anaesthetic effect}

Water quality parameters in this anaesthetic experiment are shown in Table 2. No samples died during the anaesthesia experiments due to stress from anaesthesia. Tables 3,4 , and 5 show the parameters associated with the effects of all anaesthetics at each concentration, salinity, and tem-
Table 2. Experimental water condition for river puffer, Takifugu obscurus and tiger puffer, T. rubripes in this anaesthetic experiment*

\begin{tabular}{cc}
\hline \hline Test parameters & Condition \\
\hline $\mathrm{pH}$ & $7.6 \pm 0.02$ \\
DO (dissolved oxygen; $\mathrm{mg} / \mathrm{L} ;$ & $7.5 \pm 0.32$ \\
Saturated concentration in $\left.26^{\circ} \mathrm{C}\right)$ & \\
Ammonia $(\mathrm{ppm})$ & 0.00 \\
Nitric acid $(\mathrm{ppm})$ & $1.8 \pm 0.14$ \\
Nitrous acid $(\mathrm{ppm})$ & 0.01 \\
Conductivity $(\mu \mathrm{s} / \mathrm{cm})$ & 238 \\
\hline
\end{tabular}

\footnotetext{
${ }^{*}$ Test parameters were analyzed at $1 \mathrm{hr}$ before experiment. The values are means of triplicate groups. Dissolved oxygen, $\mathrm{pH}$ and salinity were measured using an oxygen measurement electrode and a multi-data logger system (Oxyguard, Denmark). Ammonia, nitric acid, nitrous acid and conductivity were measured using spectrophotometer (DR2800, Colorado, USA).
}

perature during the test. Table 3 shows the comparison of exposure and recovery times for river puffer and tiger puffer anaesthetized under different salinities and anaesthetic concentrations. The longest exposure time was observed with 10 ppt salinity among 10, 20, and 30 ppt groups $(p<0.05)$. The shortest exposure time was observed at the intermediate salinity (20 ppt) among 10, 20, and $30 \mathrm{ppt}$, and recovery time was the longest $(p<0.05)$. However, the highest anaesthetic concentration $(60 \mathrm{ppm})$ showed no difference in exposure and recovery times $(p<0.05)$. Sea water with a $30 \mathrm{ppt}$ salinity had a lower exposure time than $10 \mathrm{ppt}$ salinity, however, it had a longer exposure time than 20 ppt salinity $(p<0.05)$. No significant differences in exposure time or recovery time were observed between the two species. When the anaesthesia concentration was increased from $15 \mathrm{ppm}$ to $30 \mathrm{ppm}$, the exposure time was drastically reduced (1.52-1.70 times lower). As the anaesthesia concentration was increased gradually from $30 \mathrm{ppm}$ to $60 \mathrm{ppm}$, the exposure time was reduced 1.34 to 1.21 
Table 3. Effects of different clove oil doses and salinity degrees on the river puffer, Takifugu obscurus and tiger puffer, T. rubripes*

\begin{tabular}{|c|c|c|c|c|c|c|c|}
\hline \multirow{3}{*}{$\begin{array}{c}\text { Salinity } \\
\text { (ppt) }\end{array}$} & \multirow{3}{*}{$\begin{array}{l}\text { Dose } \\
(\mathrm{ppm})\end{array}$} & \multicolumn{6}{|c|}{ Time $(\mathrm{sec})$} \\
\hline & & \multicolumn{3}{|c|}{ River puffer } & \multicolumn{3}{|c|}{ Tiger puffer } \\
\hline & & $\begin{array}{c}\text { Exposure } \\
\text { (E) }\end{array}$ & $\begin{array}{l}\text { Recovery } \\
\text { (R) }\end{array}$ & $\begin{array}{c}\text { Time ratio } \\
(\mathrm{sec})^{*}(\mathrm{R} / \mathrm{E})\end{array}$ & $\begin{array}{c}\text { Exposure } \\
\text { (E) }\end{array}$ & $\begin{array}{c}\text { Recovery } \\
\text { (R) }\end{array}$ & $\begin{array}{l}\text { Time ratio } \\
(\mathrm{sec})^{*}(\mathrm{R} / \mathrm{E})\end{array}$ \\
\hline \multirow{4}{*}{010} & 15 & $216.7 \pm 6.79^{\mathrm{aA}}$ & $151.5 \pm 5.46^{\mathrm{dC}}$ & $0.68 \pm 0.80^{\mathrm{cA}}$ & $210.5 \pm 5.19^{\mathrm{aA}}$ & $143.5 \pm 5.56^{\mathrm{dC}}$ & $0.68 \pm 1.19^{\mathrm{bA}}$ \\
\hline & 30 & $132.5 \pm 5.27^{\mathrm{bA}}$ & $190.3 \pm 3.61^{\mathrm{cA}}$ & $1.43 \pm 0.68^{\mathrm{bA}}$ & $128.7 \pm 4.20^{\mathrm{bA}}$ & $184.3 \pm 3.81^{\mathrm{cB}}$ & $1.43 \pm 1.30^{\mathrm{bA}}$ \\
\hline & 45 & $98.5 \pm 4.11^{\mathrm{cA}}$ & $206.5 \pm 0.70^{\mathrm{bA}}$ & $2.08 \pm 0.17^{\mathrm{bA}}$ & $96.5 \pm 3.31^{\mathrm{cA}}$ & $201.5 \pm 2.70^{b C}$ & $2.08 \pm 0.81^{\mathrm{aA}}$ \\
\hline & 60 & $79.0 \pm 2.33^{\mathrm{dA}}$ & $212.3 \pm 1.28^{\mathrm{aB}}$ & $2.68 \pm 0.54^{\mathrm{aA}}$ & $74.1 \pm 2.12^{\mathrm{dA}}$ & $214.3 \pm 2.58^{\mathrm{aB}}$ & $2.72 \pm 1.52^{\mathrm{aA}}$ \\
\hline \multirow{4}{*}{020} & 15 & $168.9 \pm 9.33^{\mathrm{aB}}$ & $176.8 \pm 6.72^{\mathrm{cA}}$ & $0.72 \pm 1.25^{\mathrm{aA}}$ & $161.3 \pm 5.01^{\mathrm{aC}}$ & $158.0 \pm 6.41^{\mathrm{dA}}$ & $0.97 \pm 1.17^{\mathrm{bA}}$ \\
\hline & 30 & $110.3 \pm 7.48^{\mathrm{bC}}$ & $186.1 \pm 5.23^{\mathrm{bB}}$ & $0.69 \pm 1.00^{\mathrm{aA}}$ & $105.5 \pm 4.53^{\mathrm{bC}}$ & $198.7 \pm 5.84^{\mathrm{cA}}$ & $1.88 \pm 1.04^{\mathrm{aA}}$ \\
\hline & 45 & $83.1 \pm 4.62^{\mathrm{cC}}$ & $190.7 \pm 3.14^{\mathrm{bB}}$ & $0.67 \pm 1.20^{\mathrm{aB}}$ & $86.3 \pm 4.50^{\mathrm{bB}}$ & $214.0 \pm 5.35^{\mathrm{aA}}$ & $2.47 \pm 1.41^{\mathrm{aA}}$ \\
\hline & 60 & $71.4 \pm 2.12^{\mathrm{dB}}$ & $206.6 \pm 2.51^{\mathrm{aC}}$ & $1.18 \pm 1.24^{\mathrm{aB}}$ & $74.8 \pm 2.21^{\mathrm{cB}}$ & $219.7 \pm 3.33^{\mathrm{bA}}$ & $2.92 \pm 1.50^{\mathrm{aA}}$ \\
\hline \multirow{4}{*}{030} & 15 & $171.7 \pm 6.76^{\mathrm{aB}}$ & $164.0 \pm 7.03^{\mathrm{dB}}$ & $0.95 \pm 1.03^{\mathrm{bA}}$ & $182.7 \pm 5.18^{\mathrm{aB}}$ & $152.0 \pm 6.06^{\mathrm{dB}}$ & $0.83 \pm 1.30^{\mathrm{bA}}$ \\
\hline & 30 & $119.6 \pm 4.38^{\mathrm{bB}}$ & $194.7 \pm 6.14^{\mathrm{cA}}$ & $1.62 \pm 1.40^{\mathrm{bA}}$ & $113.4 \pm 4.58^{\mathrm{bB}}$ & $196.3 \pm 4.89^{\mathrm{cA}}$ & $1.71 \pm 1.34^{\mathrm{aA}}$ \\
\hline & 45 & $91.4 \pm 2.95^{\mathrm{cB}}$ & $208.1 \pm 4.11^{\mathrm{bA}}$ & $2.27 \pm 1.39^{\mathrm{aA}}$ & $89.2 \pm 3.87^{\mathrm{cB}}$ & $212.1 \pm 4.26^{\mathrm{bB}}$ & $2.33 \pm 1.06^{\mathrm{aA}}$ \\
\hline & 60 & $75.1 \pm 1.84^{\mathrm{dB}}$ & $217.4 \pm 2.13^{\mathrm{aA}}$ & $2.89 \pm 1.15^{\mathrm{aA}}$ & $77.2 \pm 1.63^{\mathrm{dA}}$ & $221.4 \pm 3.03^{\mathrm{aA}}$ & $2.86 \pm 1.88^{\mathrm{aA}}$ \\
\hline
\end{tabular}

* Each value is mean $\pm \mathrm{SD}$ of triplicate experiments $(\mathrm{n}=50)$.

Different small letters indicate significant differences for the different clove oil dose at the same salinity degree $(p<0.05)$.

Different capital letters indicate significant differences for the different salinity degree at the same clove oil dose $(p<0.05)$.

times. Recovery times for both species constantly increased as the anaesthesia concentration was increased.

Table 4 shows the comparison of exposure and recovery times for two species anaesthetized with different anaesthetic concentrations and water temperatures. Overall, exposure times of both species decreased with increasing water temperature $\left(20^{\circ} \mathrm{C}\right.$ to $\left.28^{\circ} \mathrm{C}\right)$ and clove oil concentration $(p<0.05)$. Exposure times for water temperature $\left(24^{\circ} \mathrm{C}\right)$ were relatively reduced time as compared to $20^{\circ} \mathrm{C}$, on the other hand, they increased as compared to high temperature $\left(28^{\circ} \mathrm{C}\right)(p<0.05)$. Exposure times were drastically reduced with anaesthesia concentrations increasing from 15 ppm to $45 \mathrm{ppm}(1,74$ to 2,02 times lower) and with the higher concentration of $60 \mathrm{ppm}$, exposure time is shorter
(1.08 to 1.19 times shorter) at all different temperatures. Recovery times showed similar trends for all water temperatures and as concentrations increased, recovery times increased. The anaesthetic effect of the combination of clove oil and lidocaine- $\mathrm{HCl}$ is shown in Table 5. As the anaesthetic concentration was increased, exposure times tend to be reduced, and recovery times were gradually increased $(p<0.05)$. Exposure times and recovery times were not significantly different between the two species.

Water parameters of $\mathrm{DO}, \mathrm{CO}_{2}$, respiratory frequency, $\mathrm{NH}_{4}{ }^{+}$, and $\mathrm{pH}$ concentrations were measured by sedation effect of clove oil treatments. Comparison of the sedated group and the control group are shown in Tables 6 and 7 , and Fig. 1, 2, and 3. The DO, pH, and the respiratory fre- 
Table 4. Effects of different clove oil dose and water temperature on the river puffer, Takifugu obscurus and tiger puffer, T. rubripes*

\begin{tabular}{|c|c|c|c|c|c|c|c|}
\hline \multirow{3}{*}{$\begin{array}{c}\text { Tempera- } \\
\text { ture } \\
\left({ }^{\circ} \mathrm{C}\right)\end{array}$} & \multirow{3}{*}{$\begin{array}{l}\text { Dose } \\
\text { (ppm) }\end{array}$} & \multicolumn{6}{|c|}{ Time (sec) } \\
\hline & & \multicolumn{3}{|c|}{ River puffer } & \multicolumn{3}{|c|}{ Tiger puffer } \\
\hline & & $\begin{array}{l}\text { Exposure } \\
\text { (E) }\end{array}$ & $\begin{array}{l}\text { Recovery } \\
\text { (R) }\end{array}$ & $\begin{array}{l}\text { Time ratio } \\
(\mathrm{sec})^{*}(\mathrm{R} / \mathrm{E})\end{array}$ & $\begin{array}{c}\text { Exposure } \\
\text { (E) }\end{array}$ & $\begin{array}{l}\text { Recovery } \\
\text { (R) }\end{array}$ & $\begin{array}{c}\text { Time ratio } \\
(\mathrm{sec})^{*}(\mathrm{R} / \mathrm{E})\end{array}$ \\
\hline \multirow{4}{*}{20} & 15 & $164.4 \pm 6.38^{\mathrm{aA}}$ & $171.8 \pm 6.21^{\mathrm{dA}}$ & $0.84 \pm 1.01^{\mathrm{aA}}$ & $160.4 \pm 8.53^{\mathrm{aB}}$ & $167.8 \pm 8.62^{\mathrm{dA}}$ & $1.04 \pm 1.01^{\mathrm{bA}}$ \\
\hline & 30 & $125.9 \pm 5.72^{\mathrm{bA}}$ & $194.7 \pm 4.83^{\mathrm{cA}}$ & $0.86 \pm 1.15^{\mathrm{aA}}$ & $110.6 \pm 5.47^{\mathrm{bA}}$ & $203.3 \pm 6.33^{\mathrm{cA}}$ & $1.83 \pm 1.15^{\mathrm{aA}}$ \\
\hline & 45 & $86.6 \pm 3.76^{\mathrm{cA}}$ & $208.5 \pm 4.11^{\mathrm{bA}}$ & $1.09 \pm 1.58^{\mathrm{aA}}$ & $93.0 \pm 3.07^{\mathrm{cA}}$ & $211.0 \pm 4.88^{\mathrm{bA}}$ & $2.26 \pm 1.58^{\mathrm{aA}}$ \\
\hline & 60 & $79.1 \pm 1.52^{\mathrm{dA}}$ & $224.3 \pm 3.08^{\mathrm{aA}}$ & $1.36 \pm 1.63^{\mathrm{aA}}$ & $82.7 \pm 1.95^{\mathrm{cA}}$ & $219.8 \pm 3.19^{\mathrm{aA}}$ & $2.65 \pm 1.63^{\mathrm{aA}}$ \\
\hline \multirow{4}{*}{024} & 15 & $168.9 \pm 5.33^{\mathrm{aA}}$ & $175.8 \pm 5.72^{\mathrm{cA}}$ & $0.72 \pm 1.25^{\mathrm{aA}}$ & $182.7 \pm 6.18^{\mathrm{aA}}$ & $152.0 \pm 8.06^{\mathrm{dB}}$ & $0.83 \pm 1.30^{\mathrm{bA}}$ \\
\hline & 30 & $110.3 \pm 4.48^{\mathrm{bB}}$ & $184.1 \pm 4.23^{\text {bВ }}$ & $0.69 \pm 1.00^{\mathrm{aA}}$ & $113.4 \pm 4.58^{\mathrm{bA}}$ & $194.7 \pm 6.14^{\mathrm{cB}}$ & $1.71 \pm 1.34^{\mathrm{aA}}$ \\
\hline & 45 & $83.1 \pm 3.62^{\mathrm{cA}}$ & $190.7 \pm 3.14^{\mathrm{bB}}$ & $0.67 \pm 1.20^{\mathrm{aA}}$ & $89.2 \pm 3.87^{\mathrm{cA}}$ & $208.1 \pm 4.11^{\mathrm{bA}}$ & $2.33 \pm 1.06^{\mathrm{aA}}$ \\
\hline & 60 & $71.4 \pm 2.12^{\mathrm{dB}}$ & $206.6 \pm 2.51^{\mathrm{aB}}$ & $1.18 \pm 1.24^{\mathrm{aA}}$ & $77.2 \pm 1.63^{\mathrm{dB}}$ & $221.4 \pm 3.03^{\mathrm{aA}}$ & $2.86 \pm 1.88^{\mathrm{aA}}$ \\
\hline \multirow{4}{*}{28} & 15 & $138.5 \pm 4.01^{\mathrm{aB}}$ & $161.5 \pm 4.31^{\mathrm{cB}}$ & $1.04 \pm 0.96^{\mathrm{aA}}$ & $117.5 \pm 9.01^{\mathrm{aC}}$ & $155.5 \pm 6.73^{\mathrm{dB}}$ & $1.32 \pm 0.96^{\mathrm{bA}}$ \\
\hline & 30 & $90.8 \pm 3.52^{\mathrm{bC}}$ & $176.1 \pm 3.78^{\mathrm{cC}}$ & $0.86 \pm 0.75^{\mathrm{aA}}$ & $94.2 \pm 7.45^{\mathrm{bB}}$ & $187.6 \pm 4.10^{\mathrm{cC}}$ & $1.99 \pm 0.75^{\mathrm{bA}}$ \\
\hline & 45 & $79.7 \pm 3.61^{\mathrm{cB}}$ & $183.4 \pm 3.26^{\mathrm{bC}}$ & $0.90 \pm 0.82^{\mathrm{aA}}$ & $78.6 \pm 4.16^{\mathrm{cB}}$ & $191.7 \pm 3.42^{\mathrm{bB}}$ & $2.43 \pm 0.82^{\mathrm{aA}}$ \\
\hline & 60 & $66.3 \pm 1.37^{\mathrm{dC}}$ & $194.9 \pm 2.04^{\mathrm{aC}}$ & $1.48 \pm 0.60^{\mathrm{aA}}$ & $71.0 \pm 2.64^{\mathrm{dC}}$ & $198.4 \pm 1.60^{\mathrm{aB}}$ & $2.79 \pm 0.60^{\mathrm{aA}}$ \\
\hline
\end{tabular}

\footnotetext{
${ }^{*}$ Each value is mean \pm SD of triplicate experiments $(\mathrm{n}=50)$.
}

Different small letters indicate significant differences for the different clove oil dose at the same water temperature $(p<0.05)$. Different capital letters indicate significant differences for the different water temperature at the same clove oil dose $(p<0.05)$.

quency decreased up to $6 \mathrm{hrs}$, whereas the $\mathrm{NH}_{4}{ }^{+}$and $\mathrm{CO}_{2}$ concentrations increased in all groups $(p<0.05)$. Initial DO was $7.5 \pm 0.32 \mathrm{ppm}$ for all groups.

In control group, DO values for river puffer showed a significant difference after $3 \mathrm{hrs}$ and for tiger puffer after 4 hrs $(p<0.05)$, whereas in clove oil sedated group, both river puffer and tiger puffer showed no significant variation respectively (Table 6). In control group, $\mathrm{CO}_{2}$ concentrations for both river puffer and tiger puffer showed no difference, whereas in clove oil sedated group, $\mathrm{CO}_{2}$ concentrations for river puffer showed a significant variation after $5 \mathrm{hrs}$ and for tiger puffer after $3 \mathrm{hrs}(p<0.05$; Table 7$)$. Sedated group significantly showed a lower respiratory frequency $1 \mathrm{hr}$ after the experiment compared to the con- trol groups $(p<0.05)$. Also, all group for tiger puffer showed higher respiratory frequency than those of river puffer (Fig. 1). $\mathrm{NH}_{4}{ }^{+}$and $\mathrm{pH}$ values were similar to the results of DO and $\mathrm{CO}_{2}$ with being significantly different after $2 \mathrm{hrs}$ $(p<0.05$; Fig. 2 and 3$)$.

The plasma cortisol concentrations found at different times after anaesthesia are shown in Fig. 4, 5, 6, and 7. Plasma cortisol concentration at each temperature and salinity increased up to $12 \mathrm{hrs,} \mathrm{after} \mathrm{which} \mathrm{it} \mathrm{decreased}$ $(p<0.05)$. Pre-experiment plasma cortisol concentrations were less than $5 \mu \mathrm{g} / \mathrm{dL}$ in all groups $(p<0.05)$. After $1 \mathrm{hr}$, the plasma cortisol concentrations were drastically increased and the high concentrations (more than $15 \mu \mathrm{g} / \mathrm{dL}$ ) were maintained for $12 \mathrm{hrs}(p<0.05)$. After $24 \mathrm{hrs}$, concen- 
Table 5. Effects of combination of two anaesthesia mixtures of clove oil and lidocaine-HCl in river puffer, Takifugu obscurus and tiger puffer, T. rubripes*

\begin{tabular}{|c|c|c|c|c|c|c|c|}
\hline \multirow{3}{*}{$\begin{array}{l}\text { Clove oil } \\
\text { (ppm) }\end{array}$} & \multirow{3}{*}{$\begin{array}{l}\text { Lido- } \\
\text { caine- } \\
\mathrm{HCl} \\
(\mathrm{ppm})\end{array}$} & \multicolumn{6}{|c|}{ Time (sec) } \\
\hline & & \multicolumn{3}{|c|}{ River puffer } & \multicolumn{3}{|c|}{ Tiger puffer } \\
\hline & & $\begin{array}{l}\text { Exposure } \\
\text { (E) }\end{array}$ & $\begin{array}{l}\text { Recovery } \\
\text { (R) }\end{array}$ & $\begin{array}{c}\text { Time ratio } \\
(\mathrm{sec})^{*}(\mathrm{R} / \mathrm{E})\end{array}$ & $\begin{array}{c}\text { Exposure } \\
\text { (E) }\end{array}$ & $\begin{array}{l}\text { Recovery } \\
\text { (R) }\end{array}$ & $\begin{array}{c}\text { Time ratio } \\
(\mathrm{sec})^{*}(\mathrm{R} / \mathrm{E})\end{array}$ \\
\hline \multirow{3}{*}{15} & 400 & $146.0 \pm 4.41^{\mathrm{aA}}$ & $223.4 \pm 5.63^{\mathrm{cC}}$ & $1.53 \pm 1.25^{\mathrm{aB}}$ & $140.7 \pm 4.22^{\mathrm{aA}}$ & $227.5 \pm 5.37^{\mathrm{cC}}$ & $1.61 \pm 1.27^{\mathrm{bB}}$ \\
\hline & 500 & $110.1 \pm 3.84^{\mathrm{bA}}$ & $243.7 \pm 4.02^{\mathrm{bC}}$ & $2.21 \pm 1.02^{\mathrm{aB}}$ & $112.5 \pm 3.63^{\mathrm{bA}}$ & $240.3 \pm 3.81^{\mathrm{bC}}$ & $2.13 \pm 1.04^{\mathrm{aB}}$ \\
\hline & 600 & $94.6 \pm 3.11^{\mathrm{cA}}$ & $262.8 \pm 3.43^{\mathrm{aC}}$ & $2.77 \pm 1.10^{\mathrm{aC}}$ & $91.5 \pm 3.11^{\mathrm{cA}}$ & $264.5 \pm 3.07^{\mathrm{aC}}$ & $2.89 \pm 0.98^{\mathrm{aB}}$ \\
\hline \multirow{3}{*}{30} & 400 & $110.7 \pm 3.92^{\mathrm{aB}}$ & $237.1 \pm 5.04^{\mathrm{cB}}$ & $2.14 \pm 1.08^{\mathrm{bA}}$ & $117.5 \pm 4.21^{\mathrm{aB}}$ & $246.5 \pm 5.77^{\mathrm{cB}}$ & $2.09 \pm 1.46^{\mathrm{bA}}$ \\
\hline & 500 & $91.3 \pm 3.06^{\mathrm{bB}}$ & $265.5 \pm 4.26^{\mathrm{bB}}$ & $2.90 \pm 1.39^{\mathrm{aB}}$ & $96.9 \pm 3.86^{\mathrm{bB}}$ & $276.8 \pm 4.85^{\mathrm{bB}}$ & $2.85 \pm 1.25^{\mathrm{aA}}$ \\
\hline & 600 & $74.2 \pm 2.84^{\mathrm{cB}}$ & $295.4 \pm 3.88^{\mathrm{aB}}$ & $4.11 \pm 1.36^{\mathrm{aB}}$ & $78.5 \pm 3.07^{\mathrm{cB}}$ & $293.3 \pm 3.56^{\mathrm{aB}}$ & $3.79 \pm 1.15^{\mathrm{aB}}$ \\
\hline \multirow{3}{*}{45} & 400 & $89.5 \pm 3.47^{\mathrm{aC}}$ & $266.3 \pm 4.69^{\mathrm{cA}}$ & $2.97 \pm 1.35^{\mathrm{bA}}$ & $86.5 \pm 4.34^{\mathrm{aC}}$ & $257.3 \pm 6.18^{\mathrm{cA}}$ & $2.96 \pm 1.42^{\mathrm{bA}}$ \\
\hline & 500 & $70.3 \pm 2.62^{\mathrm{bC}}$ & $291.6 \pm 4.41^{\mathrm{bA}}$ & $4.21 \pm 1.68^{\mathrm{aA}}$ & $71.7 \pm 2.71^{\mathrm{bC}}$ & $294.0 \pm 5.32^{\mathrm{bA}}$ & $3.98 \pm 1.88^{\mathrm{aA}}$ \\
\hline & 600 & $58.8 \pm 2.98^{c C}$ & $319.3 \pm 3.24^{\mathrm{aA}}$ & $5.43 \pm 1.08^{\mathrm{aA}}$ & $61.6 \pm 2.16^{\mathrm{cC}}$ & $328.7 \pm 3.14^{\mathrm{aA}}$ & $5.23 \pm 1.45^{\mathrm{aA}}$ \\
\hline
\end{tabular}

${ }^{*}$ Each value is mean $\pm \mathrm{SD}$ of triplicate experiments $(\mathrm{n}=50)$.

Different small letters indicate significant differences for the different lidocaine-HCl dose at the same clove oil dose $(p<0.05)$.

Different capital letters indicate significant differences for the different clove oil dose at the same lidocaine-HCl dose $(p<0.05)$.

Table 6. Change of dissolved oxygen (DO) concentrations under the 5 ppm clove oil in river puffer, Takifugu obscurus and tiger puffer, T. rubripes at a simulated transport experiment*

\begin{tabular}{|c|c|c|c|c|}
\hline \multirow{3}{*}{$\begin{array}{l}\text { Elapsed } \\
\text { time } \\
\text { (hours) }\end{array}$} & \multicolumn{4}{|c|}{$\mathrm{DO}(\mathrm{mg} / \mathrm{L})$} \\
\hline & \multicolumn{2}{|c|}{ River puffer } & \multicolumn{2}{|c|}{ Tiger puffer } \\
\hline & Control & 5 ppm & Control & $5 \mathrm{ppm}$ \\
\hline Pre-experiment & $7.5 \pm 0.32^{\mathrm{a}}$ & $7.5 \pm 0.32^{\mathrm{a}}$ & $7.5 \pm 0.32^{\mathrm{a}}$ & $7.5 \pm 0.32^{\mathrm{a}}$ \\
\hline 1 & $6.4 \pm 0.26^{\mathrm{b}}$ & $6.9 \pm 0.24^{\mathrm{a}}$ & $6.5 \pm 0.23^{\mathrm{a}}$ & $6.7 \pm 0.25^{\mathrm{a}}$ \\
\hline 2 & $6.1 \pm 0.23^{\mathrm{a}}$ & $6.2 \pm 0.19^{\mathrm{a}}$ & $5.9 \pm 0.22^{\mathrm{a}}$ & $6.1 \pm 0.20^{\mathrm{a}}$ \\
\hline 3 & $4.9 \pm 0.24^{b}$ & $5.4 \pm 0.21^{\mathrm{a}}$ & $5.0 \pm 0.24^{\mathrm{a}}$ & $5.2 \pm 0.22^{\mathrm{a}}$ \\
\hline 4 & $4.2 \pm 0.17^{b}$ & $4.6 \pm 0.22^{\mathrm{a}}$ & $4.1 \pm 0.20^{\mathrm{b}}$ & $4.8 \pm 0.23^{\mathrm{a}}$ \\
\hline 5 & $3.7 \pm 0.18^{b}$ & $4.1 \pm 0.16^{\mathrm{a}}$ & $3.7 \pm 0.21^{b}$ & $4.1 \pm 0.19^{a}$ \\
\hline 6 & $3.3 \pm 0.20^{\mathrm{b}}$ & $3.7 \pm 0.14^{\mathrm{a}}$ & $3.5 \pm 0.19^{b}$ & $3.9 \pm 0.18^{\mathrm{a}}$ \\
\hline
\end{tabular}

\footnotetext{
${ }^{*}$ The values are means of triplicate groups $(\mathrm{n}=50)$.
}

Different small letters indicate significant differences for the different clove oil dose at same elapsed transporting time $(p<0.05)$. 
I-S Park

Table 7. Change of carbon dioxide $\left(\mathrm{CO}_{2}\right)$ concentration dose under the $5 \mathrm{ppm}$ clove oil in river puffer, Takifugu obscurus and tiger puffer, T. rubripes at a simulated transport experiment*

\begin{tabular}{|c|c|c|c|c|}
\hline \multirow{3}{*}{$\begin{array}{l}\text { Elapsed } \\
\text { time } \\
\text { (hours) }\end{array}$} & \multicolumn{4}{|c|}{$\mathrm{CO}_{2}(\mathrm{mg} / \mathrm{L})$} \\
\hline & \multicolumn{2}{|c|}{ River puffer } & \multicolumn{2}{|c|}{ Tiger puffer } \\
\hline & Control & $5 \mathrm{ppm}$ & Control & $5 \mathrm{ppm}$ \\
\hline Pre-experiment & $00.0^{\mathrm{a}}$ & $0.0^{\mathrm{a}}$ & $0.0^{\mathrm{a} 3}$ & $0.0^{\mathrm{a}}$ \\
\hline 1 & $12.1 \pm 1.89^{\mathrm{a}}$ & $11.9 \pm 2.03^{\mathrm{a}}$ & $12.5 \pm 2.14^{\mathrm{a}}$ & $11.6 \pm 1.46^{\mathrm{a}}$ \\
\hline 2 & $18.2 \pm 2.42^{\mathrm{a}}$ & $16.3 \pm 2.32^{\mathrm{a}}$ & $20.0 \pm 2.37^{\mathrm{a}}$ & $18.5 \pm 1.98^{\mathrm{a}}$ \\
\hline 3 & $27.3 \pm 2.78^{\mathrm{a}}$ & $25.7 \pm 2.84^{\mathrm{a}}$ & $29.7 \pm 2.72^{\mathrm{a}}$ & $24.3 \pm 2.38^{\mathrm{b}}$ \\
\hline 4 & $30.2 \pm 2.56^{\mathrm{a}}$ & $27.4 \pm 3.15^{\mathrm{a}}$ & $32.3 \pm 2.98^{\mathrm{a}}$ & $27.1 \pm 2.45^{\mathrm{b}}$ \\
\hline 5 & $33.8 \pm 2.08^{\mathrm{a}}$ & $28.9 \pm 2.36^{b}$ & $34.6 \pm 3.12^{\mathrm{a}}$ & $29.8 \pm 1.92^{\mathrm{b}}$ \\
\hline 6 & $35.4 \pm 2.34^{\mathrm{a}}$ & $31.3 \pm 2.52^{\mathrm{b}}$ & $37.5 \pm 2.68^{\mathrm{a}}$ & $32.7 \pm 2.96^{b}$ \\
\hline
\end{tabular}

* The values are means of triplicate groups $(\mathrm{n}=50)$.

Different small letters indicate significant differences for the different clove oil dose at the same elapsed transporting time $(p<0.05)$.
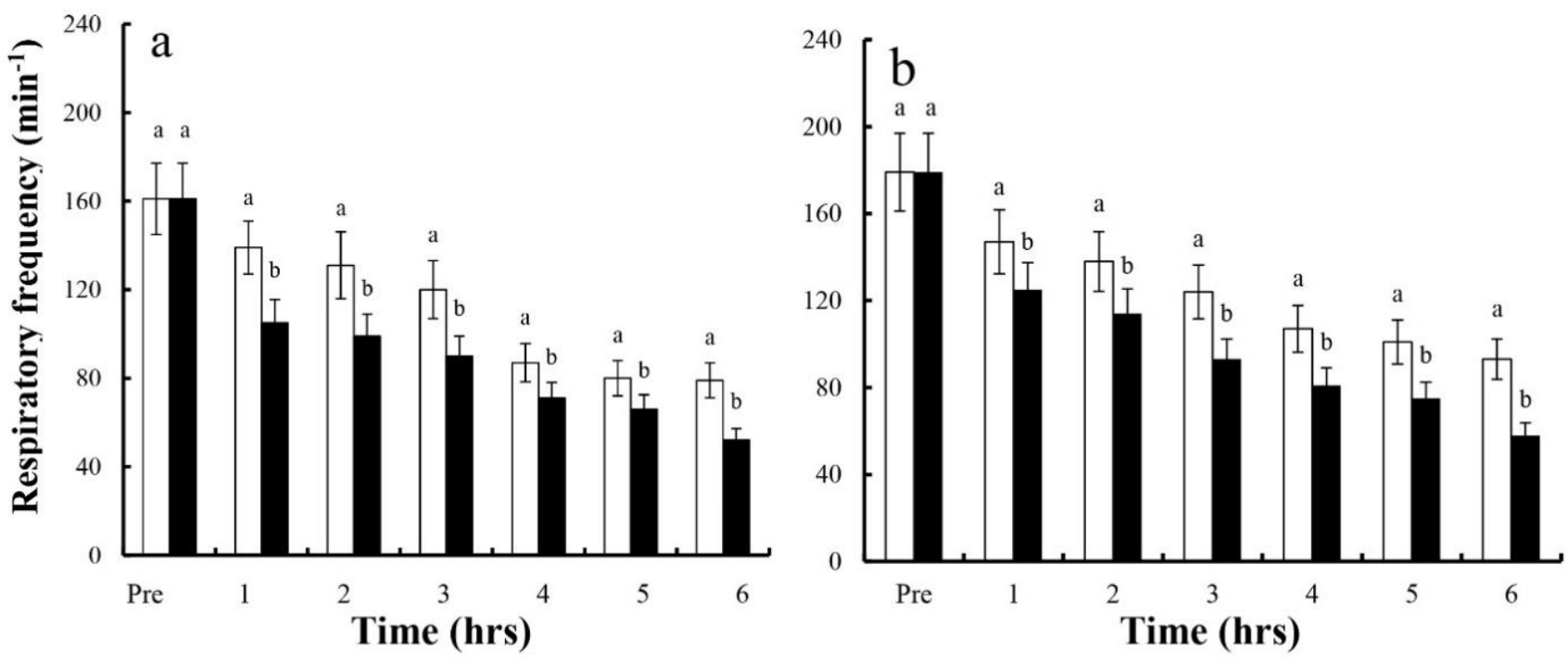

Control $\square \mathbf{p p m}$

Fig. 1. Respiratory frequency (gill cover movement) dose of clove oilin river puffer, Takifugu obscurus (a) and tiger puffer, T. rubripes (b). Values are means \pm SE of triplicate experiments $(n=50)$. Actually $n=50$ for each experiment because the means and SE were calculated separately for each group. Different letters on error bars are significantly different between no anaesthesia and clove oil anaesthesia groups $(p<0.05)$. 


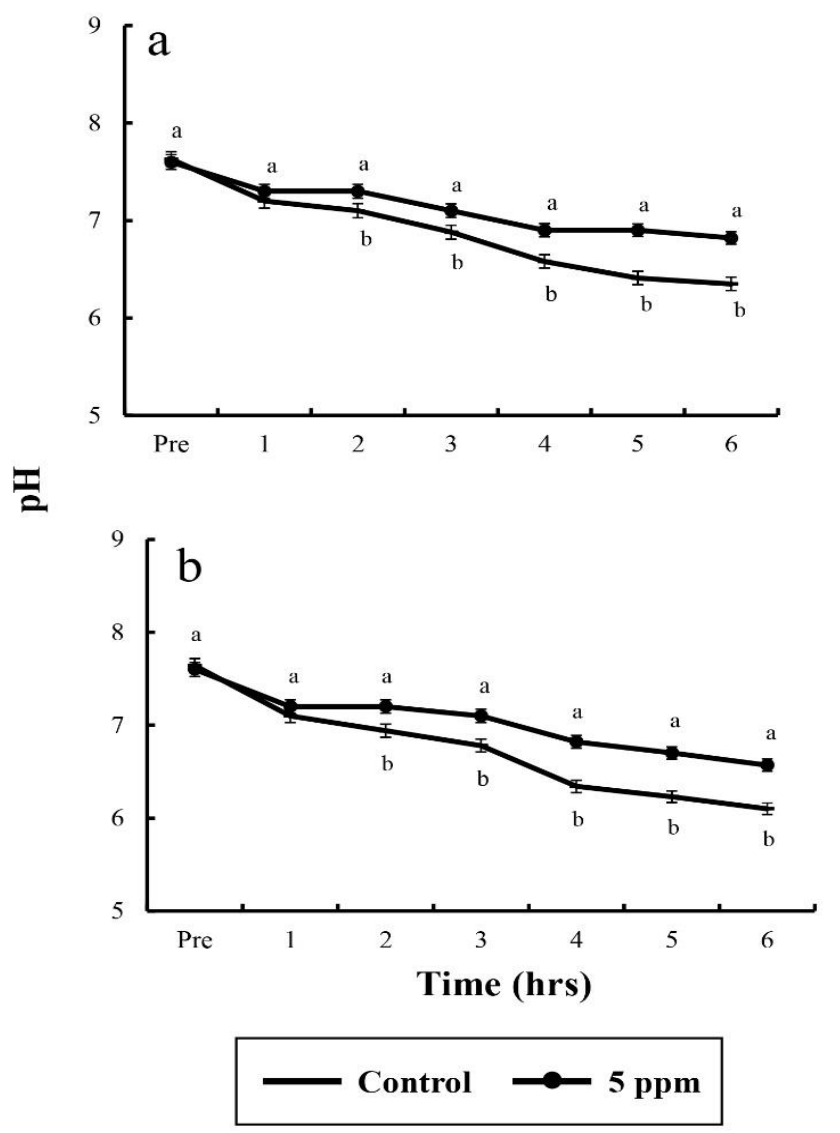

Fig. 2. pH values between salinity and dose of clove oilin river puffer, Takifugu obscurus (a) and tiger puffer, T. rubripes (b). Values are means $\pm \mathrm{SE}$ of triplicate experiments $(n=50)$. Actually $n=50$ for each experiment because the means and SE were calculated separately for each group. Different letters on error bars are significantly different between no anaesthesia and clove oil anaesthesia groups $(p<0.05)$.

trations were decreased to pre-experiment levels $(\mathrm{p}<0.05)$. At $48 \mathrm{hrs}$ after anaesthetic recovery, cortisol concentrations were almost similar to the pre-experiment values in all experimental samples.

\section{DISCUSSION}

Anaesthesia has been used to tranquilize fish regardless of their weight and standard length and it is usually an effective method for carrying out many activities such as labelling and tagging, injecting vaccines or antibiotics,

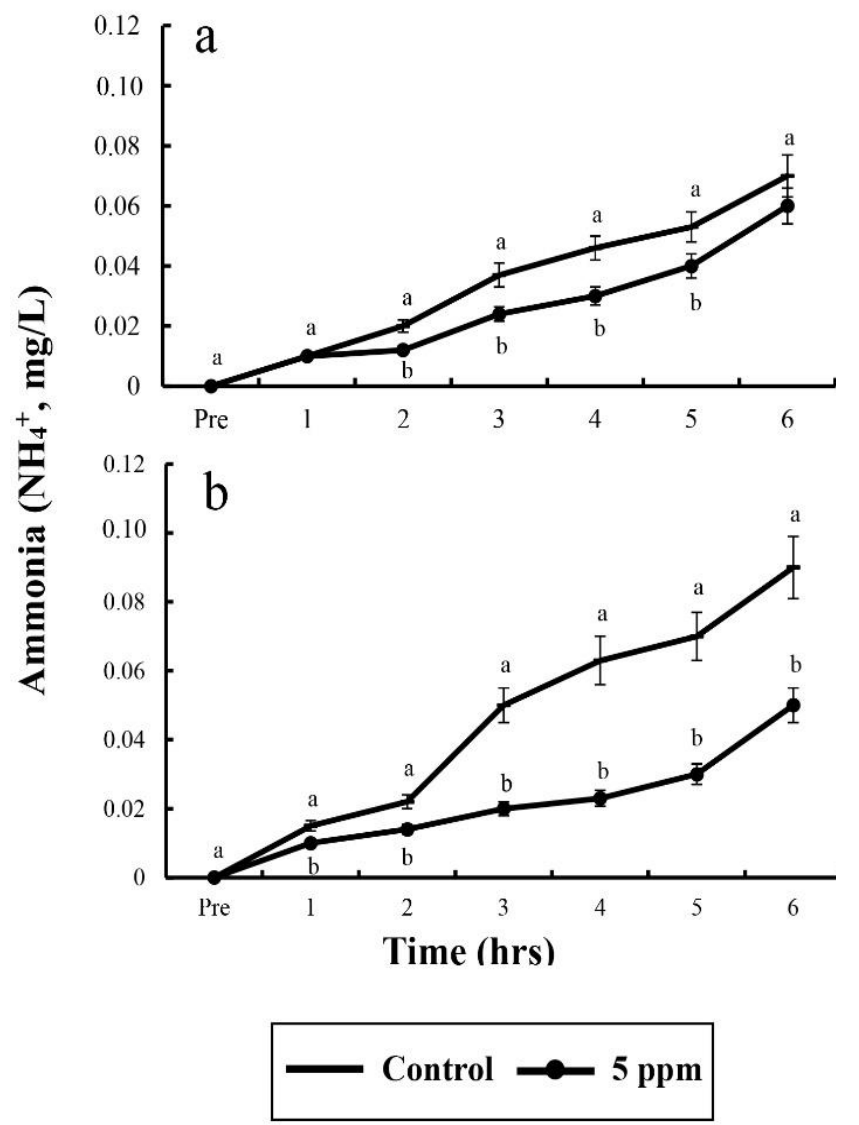

Fig. 3. Ammonia $\left(\mathrm{NH}_{4}^{+}\right)$values between salinity and dose of clove oilin river puffer, Takifugu obscurus (a) and tiger puffer, T. rubripes (b). Values are means \pm $\mathrm{SE}$ of triplicate experiments $(\mathrm{n}=50)$. Actually $\mathrm{n}=50$ for each experiment because the means and SE were calculated separately for each group. Different letters on error bars are significantly different between no anaesthesia and clove oil anaesthesia groups $(p<0.05)$.

studying physiology and ethology, photography, artificial spawning, wound healing, and live fish transport (Gil et al., 2016; Park et al., 2017a). All of these manipulations of fish contribute to the various fields of biological research (Summerfelt \& Smith, 1990; Park et al., 1998a, 1998b, 2003).

Clove oil and lidocaine- $\mathrm{HCl}$ are effective anaesthetics for fish (Bell, 1987; Park et al., 2011, 2017a, 2017b). They satisfy anaesthetic criteria including efficacy, availability, ease of usage, cost, safe for humans and environment, and 

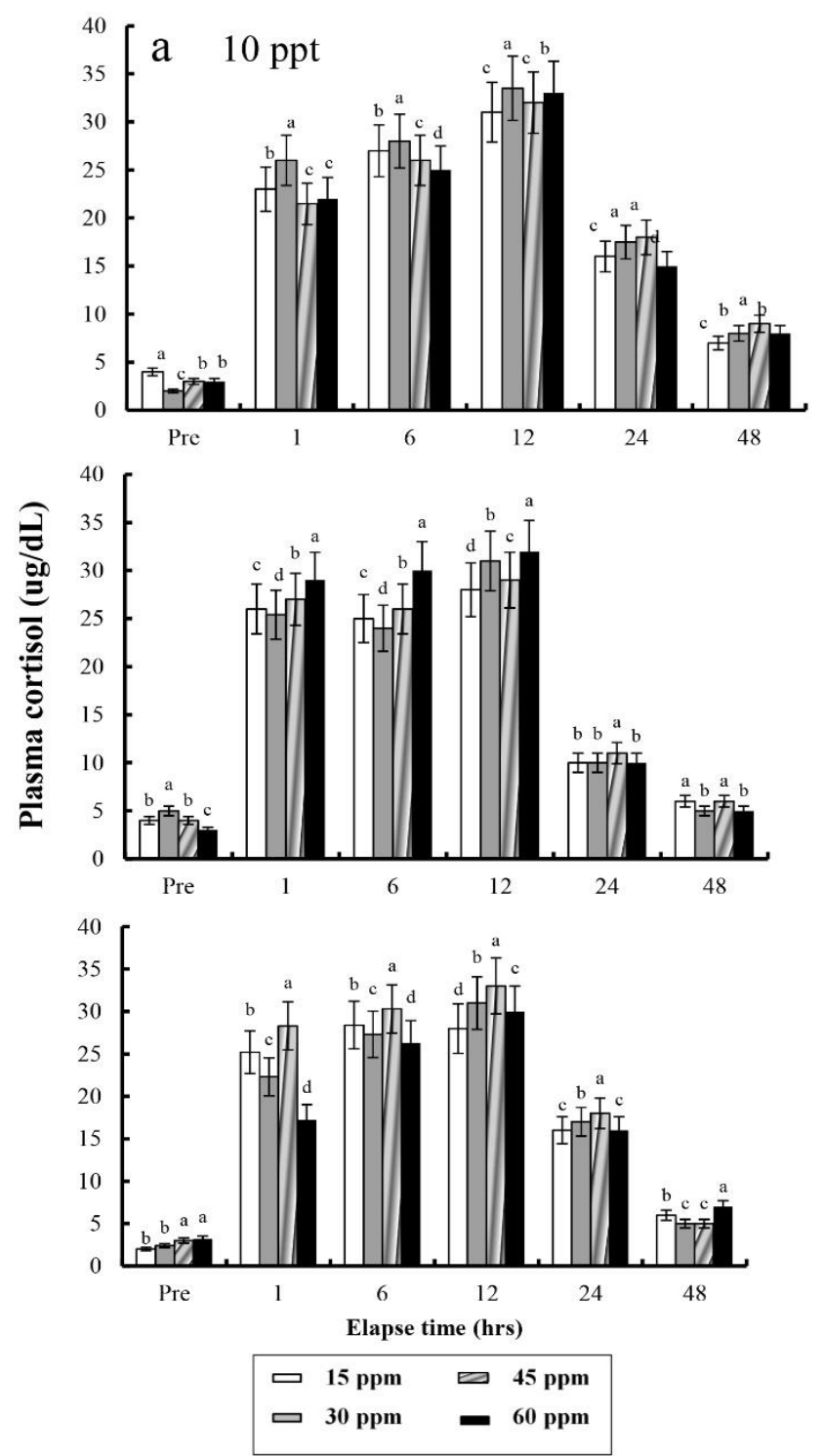

Fig. 4. Variations in the plasma cortisol concentrations in the blood plasma of the tiger puffer, Takifugu rubripes during 48 hrs of various clove oil dose and salinity in anaesthetic groups. Values are means \pm SE of triplicate experiments $(n=50)$. a: 10 ppt; b: 20 ppt; c: 30 ppt. Actually $n=50$ for each experiment because the means and SE were calculated separately for each group. Different letters on error bars are significantly different among elapsed time in anaesthesia groups $(p<0.05)$.

no fish mortality through anaesthesia. The efficacy of clove oil and lidocaine- $\mathrm{HCl}$ were tested for various fish at different doses and environmental conditions, especially
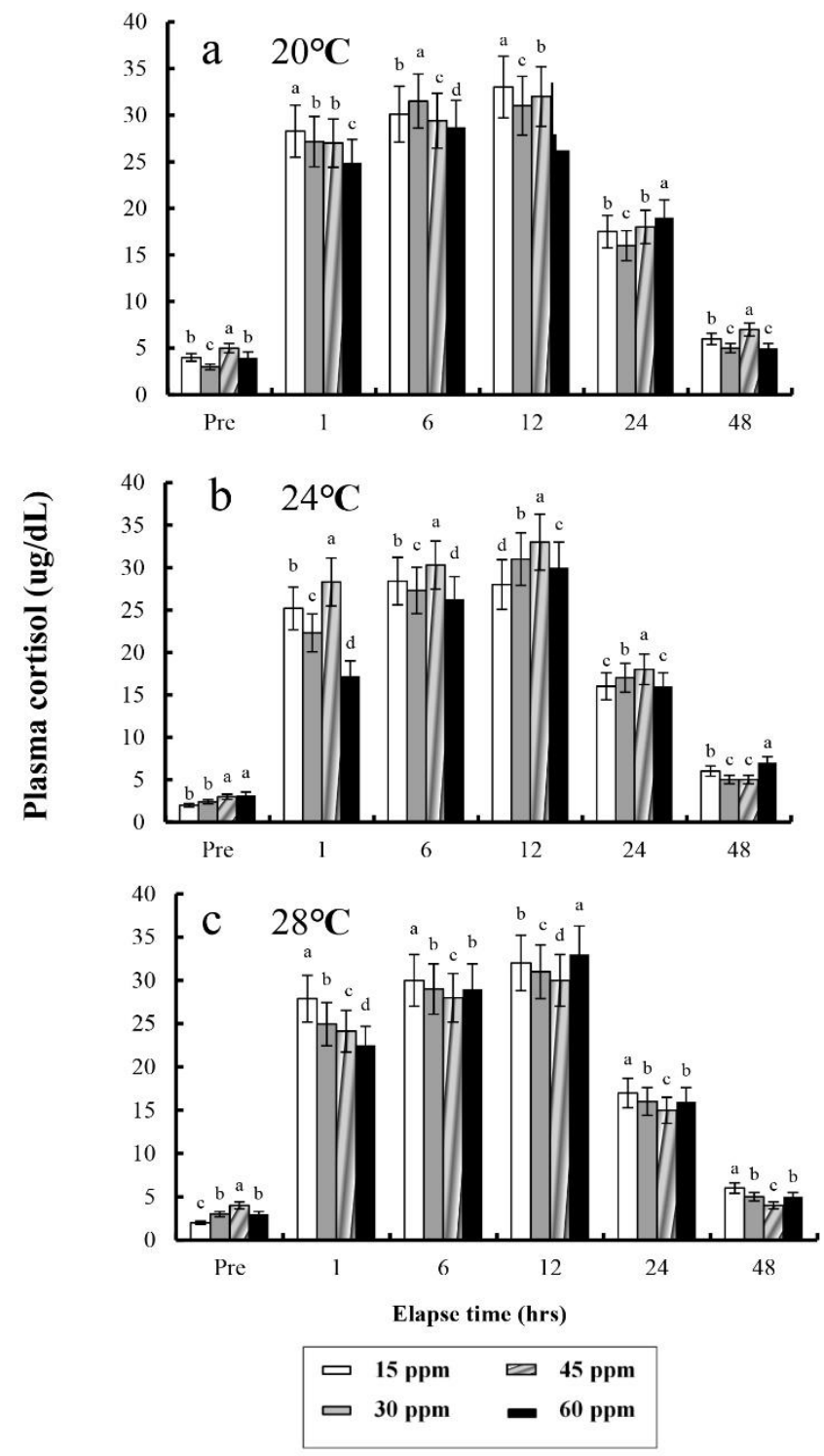

Fig. 5. Variations in the plasma cortisol concentrations in the blood plasma of the tiger puffer, Takifugu rubripes during 48 hrs of various clove oil dose and temperature in anaesthetic groups. Values are means $\pm \mathrm{SE}$ of triplicate experiments $(\mathrm{n}=50)$. a: $20^{\circ} \mathrm{C}$; b: $24^{\circ} \mathrm{C} ;$ c: $28^{\circ} \mathrm{C}$. Actually $\mathrm{n}=96$ for each experiment because the means and SE were calculated separately for each group. Different letters on error bars are significantly different among elapsed time in anaesthesia groups $(p<0.05)$.

temperatures ranging from 3 to $25^{\circ} \mathrm{C}$ and salinity ranging from 6 to 32 ppt. Metabolic activity is associated with plasma cortisol, regardless of species, the stage of life cy- 

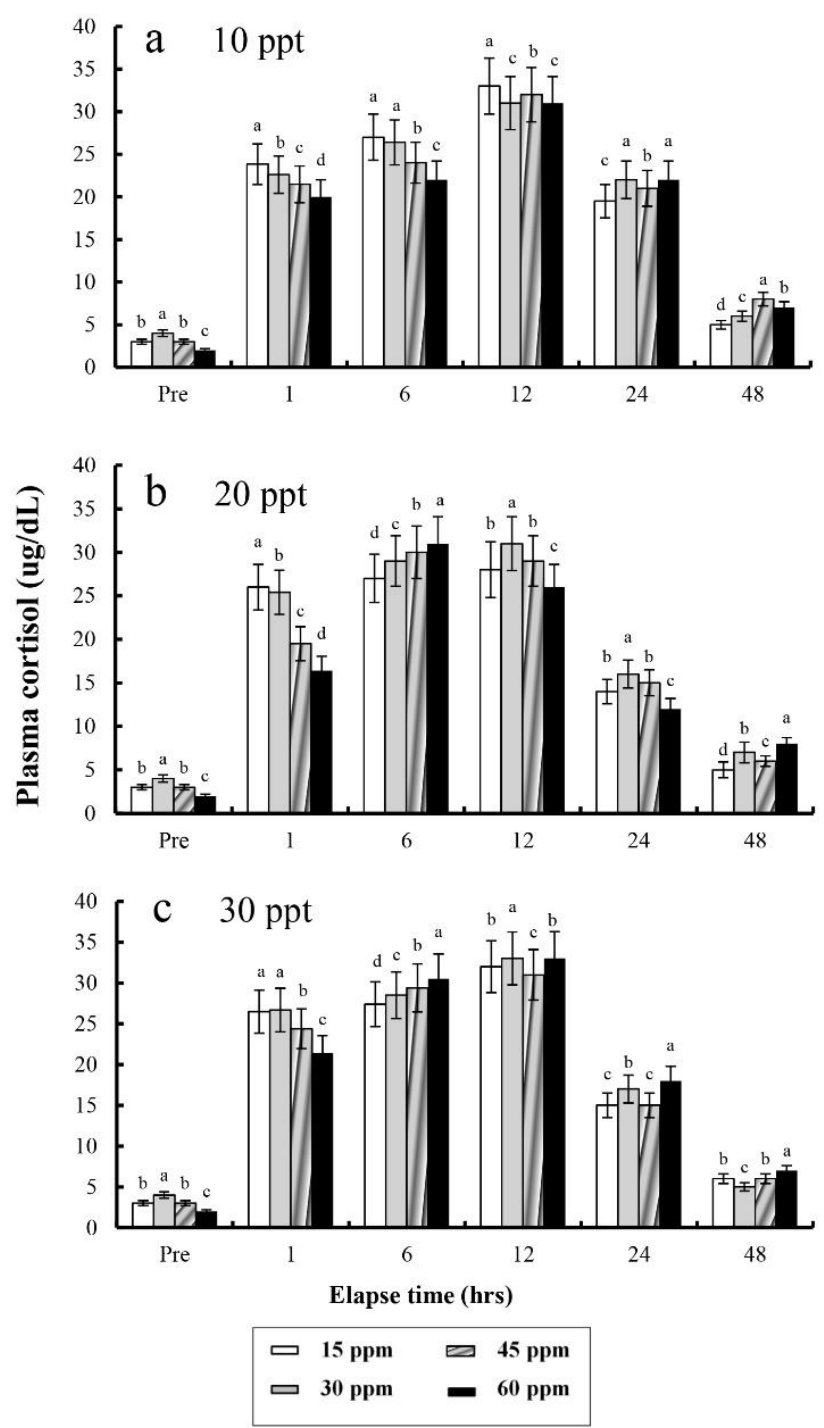

Fig. 6. Variations in the plasma cortisol concentrations in the blood plasma of the river puffer, Takifugu obscurus during 48 hrs of various clove oil dose and salinity in anaesthetic groups. Values are means \pm SE of triplicate experiments $(\mathrm{n}=50)$. a: 10 ppt; b: 20 ppt; c: 30 ppt. Actually $n=96$ for each experiment because the means and SE were calculated separately for each group. Different letters on error bars are significantly different among elapsed time in anaesthesia groups $(p<0.05)$.

cle and age, size and weight, and body condition. Plasma cor-tisol is an indicator of a stress response which is related to metabolic rate (Mommsen et al., 1999; Pirhonen \& Schreck, 2003).
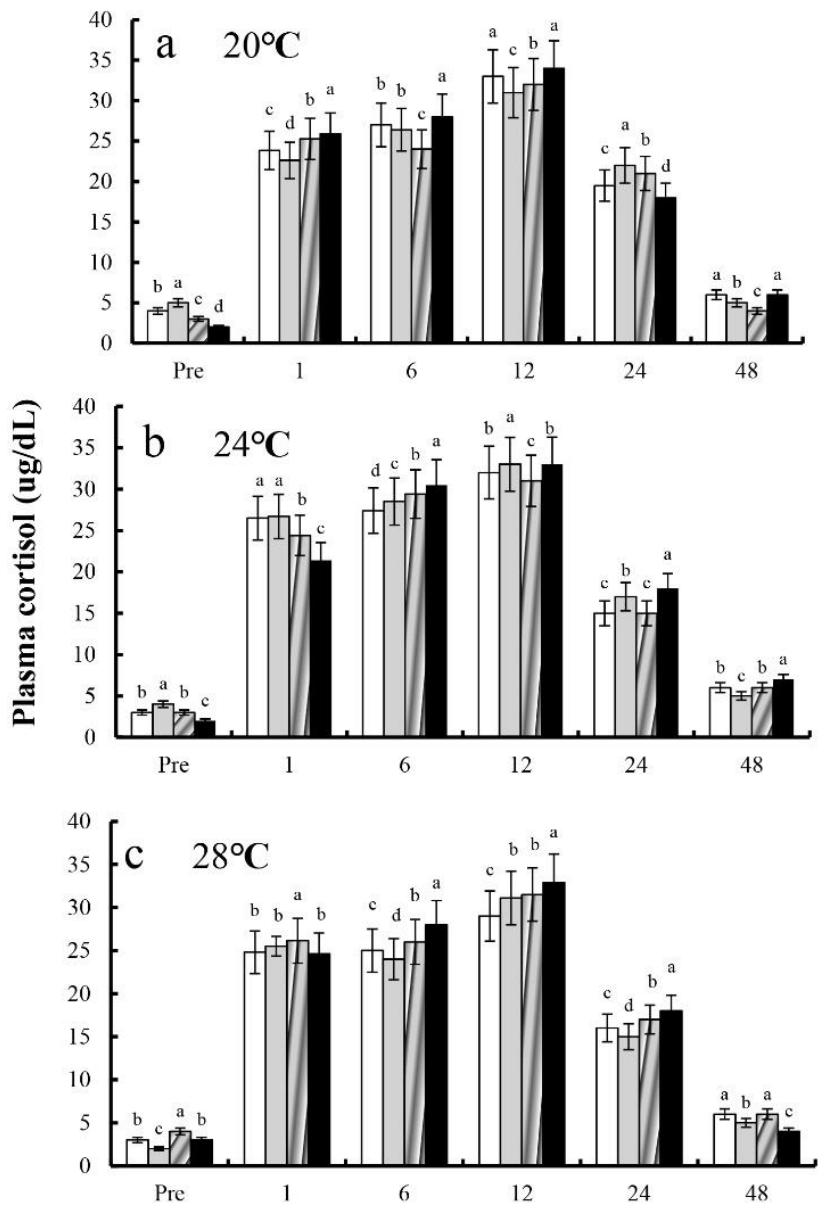

Elapse time (hrs)

$$
\begin{aligned}
& \text { 口 } 15 \mathrm{ppm} \circlearrowright 45 \mathrm{ppm} \\
& \text { 口 } 30 \mathrm{ppm}=60 \mathrm{ppm}
\end{aligned}
$$

Fig. 7. Variations in the plasma cortisol concentrations in the blood plasma of the river puffer, Takifugu obscurus during 48 hrs of various clove oil and temperature in anaesthetic groups. Values are means $\pm \mathrm{SE}$ of triplicate experiments $(\mathrm{n}=50)$. a: $20^{\circ} \mathrm{C}$; b: $24^{\circ} \mathrm{C}$; c: $28^{\circ} \mathrm{C}$. Actually $\mathrm{n}=96$ for each experiment because the means and SE were calculated separately for each group. Different letters on error bars are significantly different among elapsed time in anaesthesia groups $(p<0.05)$.

In this study, we have established the anaesthetic conditions of clove oil and clove oil - lidocaine- $\mathrm{HCl}$ combinations for river puffer and tiger puffer. Water temperature and salinity have been used to anaesthetize fish under vari- 
ous conditions, demonstrating anesthetic influence in themselves. Similar studies of changes in water temperature $\left(20^{\circ} \mathrm{C}, 24^{\circ} \mathrm{C}\right.$, and $\left.28^{\circ} \mathrm{C}\right)$, salinity $(10,20$, and $30 \mathrm{ppt})$, and anaesthesia and anaesthesia concentrations $(15,30,45$, and $60 \mathrm{ppm}$ ) were carried out in kelp grouper, Epinephelus bruneus, steelhead, Oncorhynchus mykiss, rock bream, Oplegnathus fasciatus, Caspian salmon, Salmo trutta caspius, and marine medaka, Oryzias dancena (Woolsey et al., 2004; Park et al., 2008b, 2009, 2011; Ghazilou et al., 2010). At each temperature and concentration in the anaesthesia experiment, exposure time decreased with increasing temperature and concentration and recovery time decreased with increasing temperature and concentration (Woolsey et al., 2004; Park et al., 2008b, 2009b, 2011).

Considering salinities and anaesthesia concentrations, puffer fish met the anaesthesia condition within 3 mins and recovered within 5 mins at each salinity and concentration. Ghazilou et al. (2010) studied the relation of different salinities and concentrations on exposure times and recovery times. In this study, river puffer and tiger puffer were shown to be more sensitive to clove oil, water temperature and salinity. Although the combination of two anaesthetics, clove oil and lidocaine- $\mathrm{HCl}$ in fish has not been used previously, a combination of anaesthetic agents is crucial to complement the shortcomings of using only single anaesthetic, and maximizes the benefits of the anaesthesia efficacy from the two anaesthetic agents (Schoettger \& Steucke, 1970; Sneddon, 2012). Studies of the mixture of other anaesthetics have been done before. Schoettger and Steucke (1970) studied rainbow trout, Oncorhynchus mykiss and Northern pike, Esox lucius exposed to the mixture of anaesthetics (MS-222 and quinaldine). Zahl et al. (2011) studied Atlantic halibut, Hippoglossus hippoglossus using a combination of MS-222 and benzocaine. Mixed anaesthetic agents used on rainbow trout, Northern pike, and Atlantic halibut resulted in less mortality, fewer adverse side effects, and shorter recovery times than when a single anaesthetic was used (Schoettger \& Steucke, 1970;
Zahl et al., 2011; Sneddon, 2012). Contrary to those studies, puffer fish anaesthetized with the clove oil and lidocaine- $\mathrm{HCl}$ combination showed a different tendency in recovery time. Recovery times in puffer fish were longer with combined anaesthetics (clove oil and lidocaine- $\mathrm{HCl}$ ) than with a single anaesthetic (clove oil).

There were no mortalities in both the control group and the group sedated with clove oil. During the 6-hour simulated transportation experiment, both river puffer and tiger puffer had similar trends in $\mathrm{DO}, \mathrm{CO}_{2}$, respiratory frequency, $\mathrm{NH}_{4}{ }^{+}$, and $\mathrm{pH}$, being similar to other fish species such as winter flounder, Pleuronectes americanus, Amur minnow, Rhynchocypris steindachneri, and largemouth bass, Micropterus salmoide (Park et al., 1998a, 2009a; Cooke et al., 2004). Based on these results, we can assume that the considerable variation during transportation might be caused by high stress induced by handling or netting. All water parameters $4 \mathrm{hrs}$ after experiment were similar in all experimental groups and maintained a constant values thereafter. In an observation with winter flounder, exuberant metabolic activity resulting in a rapid respiratory frequency and energy generation caused a deterioration of water quality (Park et al., 2009a).

Plasma cortisol is a useful indicator of stress in fish (Donaldson, 1981; Barton \& Iwama, 1991). Barton and Iwama (1991) stated that "Usually, the phenomenon that plasma cortisol concentration of fishes rises by stress is the first order reaction and the phenomenon that plasma glucose concentration rises is the result of second-order reaction by hormone rise reaction caused by stress". Plasma cortisol concentration did not recover to a normal level until $12 \mathrm{hrs}$ after the experiments, indicating that stress was present until $12 \mathrm{hrs}$ in this study.

In general, the main considerations in the anaesthetization of fish are safety, efficiency, price, toxicity, physiological response, and duration of withdrawal period and restriction of use (Bell, 1987; Park et al., 2003; Kang et al., 2005). Comparing the anaesthetic exposure and recovery 
times, this study shows that clove oil should be suitable for anaesthetizing river puffer and tiger puffer species. The result of this experiment suggests that safe anaesthetics such as clove oil and lidocaine- $\mathrm{HCl}$ are highly effective anaesthetic agents for puffer fish. Also, it even suggests that they could be the standard of anaesthesia for puffer fish. Still, further study in the future will have to identify more profound optimal conditions for an anesthetic combination of clove oil and licocaine- $\mathrm{HCl}$. Clove oil is a sedative which reduces the metabolic rate in transportation for a certain period of time. Clove oil is a sedative which reduces the metabolic rate in trasportation for a certain period of time. Accordingly, it may safely be said that clove oil is a highly efficient anesthetic agent as a sedative.

\section{ACKNOWLEDGEMENTS}

Comments from anonymous reviewers greatly improved the quality of the manuscript. This work was supported by a 2019 Project from Research Institute of Ocean Science and Technology (RIOST), Korea Maritime \& Ocean University, Korea. We appreciate the opportunity to undertake this study, which complied with the current laws of Korea (Ordinance of Agriculture, Food and Fisheries, No. 1 - the Law Regarding Experimental Animals, No. 9932) and the Ethical Guidelines of Korea Maritime and Ocean University, Korea.

\section{REFERENCES}

Barton BA, Iwama GK (1991) Physiological changes in fish from stress in aquaculture with emphasis on the response and effects of corticosteroids. Annu Rev Fish Dis 1:3-26.

Bell GR (1987) An outline of anesthetics and anesthesia for salmonids, a guide for fish culturists in British Columbia. Can Technic Rep Fish Aquat Sci 1534:16.

Cho GK, Heath DD (2000) Comparison of tricaine me- thanesulphonate (MS222) and clove oil anaesthesia effects on the physiology of juvenile chinook salmon Oncorhynchus tshawytscha (Walbaum). Aquacult Res 31:537-546.

Cooke SJ, Suski CD, Ostrand KG, Tufts BL, Walh DH (2004) Behavioral and physiological assessment of low concentrations of clove oil anaesthetic for handling and transporting largemouth bass (Micropterus salmoides. Aquaculture 239:509-529.

Donaldson EM (1981) The pituitary-interrenal axis as an indicator of stress in fish. In: Pickering AD (ed), Stress and Fish, Academic Press, London, pp 11-47.

Duncan DB (1955) Multiple range and multiple $F$ tests. Biometrics 11:1-42.

Ghazilou A, Hasankandi HS, Chenary F, Nateghi A, Haghi N, Sahraeean MR (2010) The anesthetic efficiency of clove oil in Caspian salmon, Salmo trutta Caspius K., Smolts in dosage--salinity-pH linked approach. J World Aquacult Soc 41:655-660.

Gil HW, Ko MG, Lee TH, Park IS, Kim DS (2016) Anesthetic effect and physiological response in olive flounder (Paralichthys olivaceus) to clove oil in a simulated transport experiment. Dev Reprod 20:255-266.

Kang BS, Go HB, Kim SJ, Na OS, Lee CH, Kim SY, Lee J, Lee YD (2005) External symptoms of tiger puffer, Takifugu rubripes infected with scuticociliates and distribution of the scuticociliates in the skin, gill and blood vessel. J Fish Pathol 18:29-37.

Kikuchi K, Furuta T, Ishizuka H, Yanagawa T (2007) Growth of tiger puffer, Takifugu rubripes, at different salinities. J World Aquacult Soc 38:427-434.

Mommsen TP, Vijayan MM, Moon TW (1999) Cortisol in teleosts: Dynamics, mechanisms of action, and metabolic regulation. Rev Fish Bio Fisher 9:211-268.

Munday PL, Wilson SK (1997) Comparative efficacy of clove oil and other chemicals in anaesthetization of Pomacentrus amboinensis, a coral reef fish. J Fish Biol 51:931-938. 
Park IS, Gil HW, Lee TH, Nam YK, Lim SG, Kim DS (2017a) Effects of clove oil and lidocaine- $\mathrm{HCl}$ anesthesia on water parameter during simulated transportation in the marine medaka, Oryzias dancena. Dev Reprod 21:19-33.

Park IS, Jo JH, Lee SJ, Kim YA, Park KE, Hur JW, Yoo JS, Song YC (2003) Anaesthetic effect of lidocaine hydrochloride-sodium bicarbonate and MS-222 on the greenling (Hexagrammos otakii). J Korean Fish Soc $36: 449-453$.

Park IS, Kim JH, Jung JB, Im JH (1998a) Effect of lidocaine as an anaesthetic on Rhynchocypris oxycephalus and R. steindachneri. J Aquacult 11:59-66.

Park IS, Lim CH, Choi MS (1998b) The evaluation of lidocaine-hydrochloride as anaesthetic for the transportation of Rhynchocypris steindachneri. J Korean Fish Soc 31:785-790.

Park IS, Lim SY, Lee TH, Gil HW, Yoo GY (2017b) Various characteristics of hybrid between river puffer, Takifugu obscurus and tiger puffer, T. rubripes, and their hybrid triploid. Dev Repord 21:181-191.

Park IS, Park MO, Hur JW, Kim DS, Chang YJ, Kim YJ, Park JY, Johnson SC (2009a) Anesthetic effects of lidocaine-hydrochloride on water parameters in simulated transport experiment of juvenile winter flounder, Pleuronectes americanus. Aquaculture 294:76-79.

Park IS, Park SJ, Gil HW, Nam YK, Kim DS (2011) Anesthetic effects of clove oil and lidocaine- $\mathrm{HCl}$ on marine medaka (Oryzias dancena). Lab Anim (NY) 40:45-51.

Park MO, Hur WJ, Im SY, Seol DW, Lee J, Park IS (2008b) Anaesthetic efficacy and physiological responses to clove oil-anaesthetized kelp grouper Epi- nephelus bruneus. Aquacult Res 39:877-884.

Park MO, Im SY, Seol DW, Park IS (2009b) Efficacy and physiological responses of rock bream, Oplegnathus fasciatus to anesthetization with clove oil. Aquaculture 287:427-430.

Pirhonen J, Schreck CB (2003) Effects of anaesthesia with MS-222, clove oil and $\mathrm{CO}_{2}$ on feed intake and plasma cortisol in steelhead trout (Oncorhynchus mykiss). Aquaculture 220:507-514.

Ross LG, Ross B (2008) Anaesthetic and Sedative Techniques for Aquatic Animals. Oxford, UK, Blackwell Science, p 236.

Schoettger RA, Steucke EW Jr (1970) Synergic mixtures of MS-222 and quinaldine as anesthetics for rainbow trout and Northern pike. Prog Fish-Cult 32:202-205.

Sneddon LU (2012) Clinical anesthesia and analgesia in fish. J Exotic Pet Med 21:32-43.

Summerfelt RC, Smith LS (1990) Anesthesia, surgery, and related techniques. In: Schreck CB, Moyle PB (eds), Methods for Fish Biology. American Fisheries Society, Bethesda, MD, pp 213-272.

Westerfield M (1993) The Zebrafish Book. Eugene, OR, University of Oregon Press, p 342.

Woolsey J, Holcomb M, Ingermann RL (2004) Effect of temperature on clove oil anesthesia in steelhead fry. North Amer J Aquacult 66:35-41.

Zahl IH, Kiessling A, Samuelsen OB, Hansen MK (2011) Anaesthesia of Atlantic halibut (Hippoglossus hippoglossus) effect of pre-anaesthetic sedation, and importance of body weight and water temperature. Aquacult Res 42:1235-1245. 